\title{
GENERALIZED LOCAL COHOMOLOGY MODULES AND HOMOLOGICAL GORENSTEIN DIMENSIONS
}

\author{
KAMRAN DIVAANI-AAZAR AND ALIREZA HAJIKARIMI
}

\begin{abstract}
Let $\mathfrak{a}$ be an ideal of a commutative Noetherian ring $R$ and $M$ and $N$ two finitely generated $R$-modules. Let $\operatorname{cd}_{\mathfrak{a}}(M, N)$ denote the supremum of the $i$ 's such that $H_{\mathfrak{a}}^{i}(M, N) \neq 0$. First, by using the theory of Gorenstein homological dimensions, we obtain several upper bounds for $\operatorname{cd}_{\mathfrak{a}}(M, N)$. Next, over a Cohen-Macaulay local ring $(R, \mathfrak{m})$, we show that

$$
\operatorname{cd}_{\mathfrak{m}}(M, N)=\operatorname{dim} R-\operatorname{grade}\left(\operatorname{Ann}_{R} N, M\right),
$$

provided that either projective dimension of $M$ or injective dimension of $N$ is finite. Finally, over such rings, we establish an analogue of the Hartshorne-Lichtenbaum Vanishing Theorem in the context of generalized local cohomology modules.
\end{abstract}

\section{INTRODUCTION}

Let $R$ be a commutative Noetherian ring with identity. The notion of generalized local cohomology was introduced by Herzog in his Habilitationsschrift [He]. Let $\mathfrak{a}$ be an ideal of $R$ and $M$ and $N$ two $R$-modules. The $i$ th generalized local cohomology module of $M$ and $N$ with respect to $\mathfrak{a}$ is defined by $H_{\mathfrak{a}}^{i}(M, N):=\underset{\vec{n}}{\lim } \operatorname{Ext}_{R}^{i}\left(M / \mathfrak{a}^{n} M, N\right)$.

Henceforth, we assume that $M$ and $N$ are finitely generated. In [B, Proposition 5.5], it is shown that the least integer $i$ such that $H_{\mathfrak{a}}^{i}(M, N) \neq 0$ is equal to grade $\left(\operatorname{Ann}_{R}(M / \mathfrak{a} M), N\right)$. We denote the supremum of $i^{\prime}$ s such that $H_{\mathfrak{a}}^{i}(M, N) \neq 0$ by $\operatorname{cd}_{\mathfrak{a}}(M, N)$ and we abbreviate $\operatorname{cd}_{\mathfrak{a}}(R, N)$ by $\operatorname{cd}_{\mathfrak{a}}(N)$. In Section 2 , we explore interrelations between generalized local cohomology modules and homological Gorenstein dimensions. This direction of research was motivated by Sazeedeh's work [Sa1], which implies that local cohomology modules can be computed by Gorenstein injective resolutions. Here, we will apply the theory of Gorenstein homological dimensions to establish the following upper bounds for $\operatorname{cd}_{\mathfrak{a}}(M, N)$ :

i) If $\operatorname{Gpd}_{N} M$ is finite, then $\operatorname{cd}_{\mathfrak{a}}(M, N) \leq \operatorname{Gpd}_{N} M+\operatorname{cd}_{\mathfrak{a}}\left(M \otimes_{R} N\right)$.

2000 Mathematics Subject Classification. 13D45, $13 \mathrm{D} 05$.

Key words and phrases. Artinian modules, attached prime ideals, cohomological dimension, generalized local cohomology modules, Gorenstein injective dimension, Gorenstein projective dimension.

The first author was supported by a grant from IPM (No. 86130114). 
ii) If $\operatorname{pd}_{N} M$ or id $N$ is finite, then $\operatorname{cd}_{\mathfrak{a}}(M, N) \leq \min \left\{\operatorname{dim} R, \operatorname{Gpd}_{N} M+\operatorname{cd}_{\mathfrak{a}}\left(M \otimes_{R}\right.\right.$ $N)\}$.

iii) If Gpd $M$ and $\operatorname{pd} N$ are finite, then $\operatorname{cd}_{\mathfrak{a}}(M, N) \leq \min \left\{\operatorname{dim} R, \operatorname{Gpd}_{N} M+\operatorname{cd}_{\mathfrak{a}}\left(M \otimes_{R}\right.\right.$ $N)\}$.

iv) If id $M$ and Gid $N$ are finite, then $\operatorname{cd}_{\mathfrak{a}}(M, N) \leq \min \left\{\operatorname{dim} R, \operatorname{Gpd}_{N} M+\operatorname{cd}_{\mathfrak{a}}\left(M \otimes_{R}\right.\right.$ $N)\}$.

v) If either $\operatorname{pd} M$ or id $M$ is finite, then $\operatorname{cd}_{\mathfrak{a}}(M, N) \leq \min \left\{\operatorname{Gid} N, \operatorname{Gpd}_{N} M+\operatorname{cd}_{\mathfrak{a}}\left(M \otimes_{R}\right.\right.$ $N)\}$.

Here Gid and Gpd stand for Gorenstein injective and projective dimensions, respectively. Also $\operatorname{pd}_{N} M:=\sup \left\{\operatorname{pd}_{R_{\mathfrak{p}}} M_{\mathfrak{p}}: \mathfrak{p} \in \operatorname{Supp}_{R} M \cap \operatorname{Supp}_{R} N\right\}$ and $\operatorname{Gpd}_{N} M:=\sup \left\{i \in \mathbb{N}_{0}:\right.$ $\left.\operatorname{Ext}_{R}^{i}(M, N) \neq 0\right\}$, with the usual convention that the supremum of the empty set of integers is interpreted as $-\infty$. As an application of these bounds, one can improves [HZ, Theorem 3.2] and Theorem 3.1 and Lemma 5.4 in [CH], see Corollary 2.6 below.

Let $(R, \mathfrak{m}, k)$ be a local ring. Grothendieck's non-Vanishing Theorem implies that $\operatorname{cd}_{\mathfrak{m}}(N)=\operatorname{dim} N$. But, not much is known about $\operatorname{cd}_{\mathfrak{m}}(M, N)$. In [HZ], the class of finitely generated $R$-modules $L$ for which $\operatorname{cd}_{\mathfrak{m}}(L, N)=\operatorname{depth} N$ is investigated. In this paper, we intend to compute $\mathrm{cd}_{\mathfrak{m}}(M, N)$, when either projective dimension of $M$ or injective dimension of $N$ is finite. Note that we have to impose these assumptions on $M$ or $N$, because otherwise $\operatorname{cd}_{\mathfrak{m}}(M, N)$ might be infinite. To realize an easy example, suppose that $M$ has infinite projective dimension. Then $H_{\mathfrak{m}}^{i}(M, k) \cong \operatorname{Ext}_{R}^{i}(M, k)$ is non-zero for infinitely many $i$. Now, let $R$ be Cohen-Macaulay and suppose that either projective dimension of $M$ or injective dimension of $N$ is finite. In Section 3, we show that

$$
\operatorname{cd}_{\mathfrak{m}}(M, N)=\operatorname{dim} R-\operatorname{grade}\left(\operatorname{Ann}_{R} N, M\right) .
$$

Next, we show that $H_{\mathfrak{a}}^{d}(M, N), d:=\operatorname{dim} R$, can be described as a certain quotient of $H_{\mathfrak{m}}^{d}(M, N)$, see Theorem 3.8 below. Then as an immediate application, we will compute the set of attached prime ideals of the Artinian $R$-module $H_{\mathfrak{a}}^{d}(M, N)$. More precisely, we show that

$$
\operatorname{Att}_{R}\left(H_{\mathfrak{a}}^{d}(M, N)\right)=\left\{\mathfrak{p} \cap R: \mathfrak{p} \in \operatorname{Supp}_{\hat{R}} \hat{N} \cap \operatorname{Ass}_{\hat{R}} \hat{M} \text { and } \operatorname{dim}(\hat{R} / \mathfrak{a} \hat{R}+\mathfrak{p})=0\right\} .
$$

This enables us to establish an analogue of the Hartshorne-Lichtenbaum Vanishing Theorem for generalized local cohomology modules to the effect that the following are equivalent.

i) $H_{\mathfrak{a}}^{d}(M, N)=0$.

ii) $H_{\mathfrak{m}}^{d}(M, N)=\sum_{n \in \mathbb{N}}<\mathfrak{m}>\left(0:_{H_{\mathfrak{m}}^{d}(M, N)} \mathfrak{a}^{n}\right)$.

iii) For any integer $l \in \mathbb{N}$, there exists an $n=n(l) \in \mathbb{N}$ such that

$$
0:_{H_{\mathfrak{m}}^{d}(M, N)} \mathfrak{a}^{l} \subseteq<\mathfrak{m}>\left(0:_{H_{\mathfrak{m}}^{d}(M, N)} \mathfrak{a}^{n}\right) .
$$


iv) $\operatorname{dim} \hat{R} / \mathfrak{a} \hat{R}+\mathfrak{p}>0$ for all $\mathfrak{p} \in \operatorname{Supp}_{\hat{R}} \hat{N} \cap \operatorname{Ass}_{\hat{R}} \hat{M}$.

(Here for an Artinian $R$-module $A$, we use $<\mathfrak{m}>A$ for denoting the submodule $\underset{i \in \mathbb{N}}{\cap} \mathfrak{m}^{i} A$.)

2. SOME UPPER BOUNDS FOR $\mathrm{cd}_{\mathfrak{a}}(M, N)$

We start this section by introducing the notions of relative projective dimension and of relative Gorenstein projective dimension for a pair of finitely generated $R$-modules. But, first let recall some definitions from the theory of Gorenstein homological dimensions. The theory of Gorenstein homological dimensions was initiated by Enochs and Jenda in 1995, see [EJ]. An $R$-module $N$ is said to be Gorenstein injective if there exists an exact sequence

$$
I^{\bullet}: \cdots \longrightarrow I_{1} \longrightarrow I_{0} \longrightarrow I^{0} \longrightarrow I^{1} \longrightarrow \cdots
$$

of injective $R$-modules such that $N \cong \operatorname{ker}\left(I^{0} \longrightarrow I^{1}\right)$ and that $\operatorname{Hom}_{R}\left(I, I^{\bullet}\right)$ is exact for all injective $R$-modules $I$. Also, an $R$-module $N$ is said to be Gorenstein projective if there exists an exact sequence

$$
P_{\bullet}: \cdots \longrightarrow P_{1} \longrightarrow P_{0} \longrightarrow P^{0} \longrightarrow P^{1} \longrightarrow \cdots
$$

of projective $R$-modules such that $N \cong \operatorname{ker}\left(P^{0} \longrightarrow P^{1}\right)$ and that $\operatorname{Hom}_{R}\left(P_{\bullet}, P\right)$ is exact for all projective $R$-modules $P$. Then for an $R$-module $N$, the notion Gid $N$, Gorenstein injective dimension of $N$, is defined as the infimum of the length of right resolutions of $N$ which are consisting of Gorenstein injective modules. Similarly, Gpd N, Gorenstein projective dimension of $N$, is defined as the infimum of the length of left resolutions of $N$ which are consisting of Gorenstein projective modules. It is known that if id $N$ (, respectively $\operatorname{pd} N$ ) is finite, then Gid $N=$ id $N($, respectively Gpd $N=\operatorname{pd} N$ ).

By [Ho, Corollary 2.21], if Gpd $M<\infty$, then $\operatorname{Gpd} M=\sup \left\{i \in \mathbb{N}_{0}: \operatorname{Ext}_{R}^{i}(M, R) \neq 0\right\}$. In view of this, our second definition below might seem to be rational.

Definition 2.1. Let $M$ and $N$ be two finitely generated $R$-modules. We define projective dimension of $M$ relative to $N$ by

$$
\operatorname{pd}_{N} M:=\sup \left\{\operatorname{pd}_{R_{\mathfrak{p}}} M_{\mathfrak{p}}: \mathfrak{p} \in \operatorname{Supp}_{R} M \cap \operatorname{Supp}_{R} N\right\} .
$$

Also, we define Gorenstein projective dimension of $M$ relative to $N$ by

$$
\operatorname{Gpd}_{N} M:=\sup \left\{i \in \mathbb{N}_{0}: \operatorname{Ext}_{R}^{i}(M, N) \neq 0\right\} .
$$

Note that the above mentioned result of Holm indicates that if $\operatorname{Gpd} M<\infty$, then $\operatorname{Gpd}_{R} M=\operatorname{Gpd} M$. For sake of completeness, we collect all needed properties of these newly defined notions in a lemma.

Lemma 2.2. Let $M$ and $N$ be two non-zero finitely generated R-modules.

i) $\operatorname{grade}\left(\operatorname{Ann}_{R} M, N\right) \leq \operatorname{Gpd}_{N} M$. 
ii) $\operatorname{pd}_{N} M \leq \operatorname{pd} M$ and if $R$ is local, then $\operatorname{pd}_{N} M=\operatorname{pd} M$.

iii) $\operatorname{Gpd}_{N} M \leq \min \{\operatorname{pd} M, \mathrm{id} N\}$. In particular, if either $\operatorname{pd} M$ or id $N$ is finite, then $\operatorname{Gpd}_{N} M$ is finite.

iv) If $\mathrm{pd}_{N} M$ is finite, then $\operatorname{Gpd}_{N} M=\mathrm{pd}_{N} M$. In particular, if $R$ is local and $\mathrm{pd} M$ is finite, then $\mathrm{Gpd}_{N} M=\mathrm{pd} M$.

v) If $\mathrm{Gpd} M$ and $\mathrm{pd} N$ are finite, then $\mathrm{Gpd}_{N} M \leq \mathrm{Gpd} M$.

vi) If id $M$ and Gid $N$ are finite, then $\operatorname{Gpd}_{N} M \leq \operatorname{Gid} N$.

vii) If $\operatorname{pd}_{N} M$ is finite, then $\bigcup_{i \in \mathbb{N}_{0}} \operatorname{Supp}_{R}\left(\operatorname{Ext}_{R}^{i}(M, N)\right)=\operatorname{Supp}_{R}\left(M \otimes_{R} N\right)$, and so $\operatorname{dim}\left(M \otimes_{R} N\right)=\max \left\{\operatorname{dim} \operatorname{Ext}_{R}^{i}(M, N): i \in \mathbb{N}_{0}\right\}$.

viii) If $\operatorname{pd}_{N} M$ is finite, then for any ideal $\mathfrak{a}$ of $R, \operatorname{cd}_{\mathfrak{a}}\left(M \otimes_{R} N\right)=\max \left\{\operatorname{cd}_{\mathfrak{a}}\left(\operatorname{Ext}_{R}^{i}(M, N)\right)\right.$ : $\left.i \in \mathbb{N}_{0}\right\}$.

ix) If $R$ is local and id $N<\infty$, then $\operatorname{Gpd}_{N} M=\operatorname{depth} R-\operatorname{depth} M$. In addition, if $M$ is maximal Cohen-Macaulay, then $\operatorname{Gpd}_{N} M=0$.

x) If id $N<\infty$, then $\operatorname{Gpd}_{N} M=\sup \left\{\right.$ htp $\left.-\operatorname{depth} M_{\mathfrak{p}}: \mathfrak{p} \in \operatorname{Supp}_{R} M \cap \operatorname{Supp}_{R} N\right\}$.

xi) $\operatorname{id}_{R_{\mathfrak{p}}} N_{\mathfrak{p}}<\infty$ for all prime ideals $\mathfrak{p}$ of $R$ if and only if $\operatorname{Gpd}_{N} L<\infty$ for all finitely generated $R$-modules $L$.

Proof. i), ii) and iii) follow immediately by the definitions.

iv) Let $i>\operatorname{pd}_{N} M$. Then for any $\mathfrak{p} \in \operatorname{Supp}_{R} M \cap \operatorname{Supp}_{R} N$, it turns out that

$$
\operatorname{Ext}_{R}^{i}(M, N)_{\mathfrak{p}} \cong \operatorname{Ext}_{R_{\mathfrak{p}}}^{i}\left(M_{\mathfrak{p}}, N_{\mathfrak{p}}\right)=0,
$$

as $i>\operatorname{pd}_{R_{\mathfrak{p}}} M_{\mathfrak{p}}$. Hence $\operatorname{Gpd}_{N} M \leq \mathrm{pd}_{N} M$.

Over a local ring $T$, for any two non-zero finitely generated $T$-modules $M$ and $L$, [Mat, page 154, Lemma 1] implies that if $\operatorname{pd} M<\infty$, then $\operatorname{Gpd}_{L} M=\operatorname{pd} M$. Let $\mathfrak{p} \in \operatorname{Supp}_{R} M \cap$ $\operatorname{Supp}_{R} N$ and $h:=\operatorname{pd}_{R_{\mathfrak{p}}} M_{\mathfrak{p}}$. Then

$$
\operatorname{Ext}_{R}^{h}(M, N)_{\mathfrak{p}} \cong \operatorname{Ext}_{R_{\mathfrak{p}}}^{h}\left(M_{\mathfrak{p}}, N_{\mathfrak{p}}\right) \neq 0,
$$

and so $\mathrm{pd}_{R_{\mathfrak{p}}} M_{\mathfrak{p}} \leq \operatorname{Gpd}_{N} M$. Therefore $\operatorname{Gpd}_{N} M=\operatorname{pd}_{N} M$.

v) and vi) follow by [Ho, Theorem 2.20] and [Ho, Theorem 2.22], respectively.

vii) It is obvious that each module $\operatorname{Ext}_{R}^{i}(M, N)$ is supported in $\operatorname{Supp}_{R} M \cap \operatorname{Supp}_{R} N=$ $\operatorname{Supp}_{R}\left(M \otimes_{R} N\right)$. Now, let $\mathfrak{p} \in \operatorname{Supp}_{R} M \cap \operatorname{Supp}_{R} N$ and set $h:=\operatorname{pd}_{R_{\mathfrak{p}}} M_{\mathfrak{p}}$. We observed earlier in the proof of part iv) that $\operatorname{Ext}_{R_{\mathfrak{p}}}^{h}\left(M_{\mathfrak{p}}, N_{\mathfrak{p}}\right) \neq 0$. Hence $\mathfrak{p} \in \operatorname{Supp}_{R}\left(\operatorname{Ext}_{R}^{h}(M, N)\right)$, and the conclusion follows.

viii) For any two finitely generated $R$-modules $X$ and $Y$ with $\operatorname{Supp}_{R} X \subseteq \operatorname{Supp}_{R} Y$, [DNT, Theorem 2.2] implies that $\operatorname{cd}_{\mathfrak{a}}(X) \leq \operatorname{cd}_{\mathfrak{a}}(Y)$. Let $Z$ be a finitely generated $R$ module. Since $Z$ and $\oplus_{\mathfrak{p} \in \operatorname{Ass}_{R} Z} R / \mathfrak{p}$ have the same support, it follows that

$$
\operatorname{cd}_{\mathfrak{a}}(Z)=\sup \left\{\operatorname{cd}_{\mathfrak{a}}(R / \mathfrak{p}): \mathfrak{p} \in \operatorname{Supp}_{R} Z\right\} .
$$

Now, the claim becomes clear in light of part vii). 
ix) By [I, 2.6], it follows that $\sup \left\{i \in \mathbb{N}_{0}: \operatorname{Ext}_{R}^{i}(M, N) \neq 0\right\}=\operatorname{depth} R-\operatorname{depth} M$.

x) Let $\mathfrak{p} \in \operatorname{Supp}_{R} N$. Since $\operatorname{id}_{R} N<\infty$, it turns out that $\operatorname{id}_{R_{\mathfrak{p}}} N_{\mathfrak{p}}<\infty$, and so $R_{\mathfrak{p}}$ is Cohen-Macaulay. Hence, it follows by ix) that

$$
\begin{aligned}
\operatorname{Gpd}_{N} M & =\sup \left\{\operatorname{Gpd}_{N_{\mathfrak{p}}} M_{\mathfrak{p}}: \mathfrak{p} \in \operatorname{Supp}_{R} M \cap \operatorname{Supp}_{R} N\right\} \\
& =\sup \left\{\text { ht } \mathfrak{p}-\operatorname{depth}_{R_{\mathfrak{p}}} M_{\mathfrak{p}}: \mathfrak{p} \in \operatorname{Supp}_{R} M \cap \operatorname{Supp}_{R} N\right\} .
\end{aligned}
$$

xi) is the main result of [T].

Example 2.3. There exist a non-Gorenstein Artinian local ring $(R, \mathfrak{m})$ and a finitely generated $R$-module $M$, which is not Gorenstein projective such that $\operatorname{Hom}_{R}(M, R) \neq 0$ and $\operatorname{Ext}_{R}^{i}(M, R)=0$ for all $i>0$. So, although neither projective dimension of $M$ nor injective dimension of $R$ is finite, one has $\operatorname{Gpd}_{R} M=0<\infty$. For a concrete realization of this example, we refer the reader to [JS, Theorem 1.7].

We need the following lemma in the proof of Theorem 2.5 and Corollary 2.6 below. It generalizes [HZ, Lemma 3.1].

Lemma 2.4. Assume that $M$ and $N$ are non-zero finitely generated $R$-modules such that $\operatorname{Gpd}_{N} M<\infty$. Then obviously $\operatorname{dim} \operatorname{Ext}_{R}^{i}(M, N) \leq \operatorname{dim}\left(M \otimes_{R} N\right)+\operatorname{Gpd}_{N} M-i$ for all i. Moreover, if the assumption $\operatorname{Gpd}_{N} M<\infty$ replaced by any of the following stronger assumptions, then $\operatorname{dim} \operatorname{Ext}_{R}^{i}(M, N) \leq \min \left\{\operatorname{dim} R, \operatorname{dim}\left(M \otimes_{R} N\right)+\operatorname{Gpd}_{N} M\right\}-i$ for all $i$.

i) Gpd $M$ and pd $N$ are finite.

ii) id $M$ and Gid $N$ are finite.

iii) id $N$ is finite.

iv) $\operatorname{pd}_{N} M$ is finite.

Proof. Let $i \in \mathbb{N}_{0}$. If $\operatorname{Ext}_{R}^{i}(M, N)=0$, then there is nothing to prove. So, we may assume that $i \leq \operatorname{Gpd}_{N} M$. On the other hand, $\operatorname{dim} \operatorname{Ext}_{R}^{i}(M, N) \leq \operatorname{dim}\left(M \otimes_{R} N\right)$, since $\operatorname{Ext}_{R}^{i}(M, N)$ is supported in $\operatorname{Supp}_{R}\left(M \otimes_{R} N\right)$. Hence $\operatorname{dim} \operatorname{Ext}_{R}^{i}(M, N)+i \leq \operatorname{dim}\left(M \otimes_{R}\right.$ $N)+\operatorname{Gpd}_{N} M$.

For a while, we assume that $R$ is local. By [AB, 3.7], if Gpd $M<\infty$, then Gpd $M=$ $\operatorname{depth} R-\operatorname{depth} M$. By [KTY] (, respectively [Mat, Theorem 18.9]), if Gid N (, respectively id $N$ ) is finite, then Gid $N$ (, respectively id $N$ ) is equal to depth $R$. Also, by the AuslanderBuchsbaum formula if pd $M<\infty$, then

$$
\operatorname{Gpd}_{N} M=\operatorname{pd} M=\operatorname{depth} R-\operatorname{depth} M .
$$

Hence in view of Lemma 2.2, each of the conditions i), ii), iii) and iv) implies that $\operatorname{Gpd}_{N} M \leq \operatorname{depth} R$. But for any $\mathfrak{p} \in \operatorname{Supp}_{R} M \cap \operatorname{Supp}_{R} N$, all of the conditions i), ii), iii) and iv) are preserved under localization at $\mathfrak{p}$. Thus, for any such prime ideal $\mathfrak{p}$, each of the conditions i), ii), iii) and iv) implies that $\operatorname{Gpd}_{N_{\mathfrak{p}}} M_{\mathfrak{p}} \leq \mathrm{htp}$. 
Assume one of the conditions i), ii), iii) and iv) holds and let $\mathfrak{p} \in \operatorname{Supp}_{R}\left(\operatorname{Ext}_{R}^{i}(M, N)\right)$. Clearly, we can suppose that $\operatorname{dim} R$ is finite. Then we have $i \leq \operatorname{Gpd}_{N_{\mathfrak{p}}} M_{\mathfrak{p}} \leq \mathrm{htp}$, and so $\operatorname{dim} R / \mathfrak{p} \leq \operatorname{dim} R-$ ht $\mathfrak{p} \leq \operatorname{dim} R-i$. Thus

$$
\operatorname{dim} \operatorname{Ext}_{R}^{i}(M, N)=\sup \left\{\operatorname{dim} R / \mathfrak{p}: \mathfrak{p} \in \operatorname{Supp}_{R}\left(\operatorname{Ext}_{R}^{i}(M, N)\right)\right\} \leq \operatorname{dim} R-i .
$$

Now, in view of the first assertion, the proof is complete.

Let $\mathfrak{a}$ be an ideal of $R$ and $M$ a finitely generated $R$-module of finite dimension d. It is known that $H_{\mathfrak{a}}^{d}(M)$ is Artinian. Also, by [Mar, Corollary 2.5], we know that $\operatorname{Supp}_{R} H_{\mathfrak{a}}^{d-1}(M)$ is finite. The second assertion of the following result might be considered as a generalization of these facts to generalized local cohomology modules.

Theorem 2.5. Let $\mathfrak{a}$ be an ideal of $R$ and $M$ and $N$ two finitely generated $R$-modules such that $p:=\operatorname{Gpd}_{N} M<\infty$ and set $c:=\operatorname{Gpd}_{N} M+\operatorname{cd}_{\mathfrak{a}}\left(M \otimes_{R} N\right)$. Then $H_{\mathfrak{a}}^{c}(M, N) \cong$ $H_{\mathfrak{a}}^{c-p}\left(\operatorname{Ext}_{R}^{p}(M, N)\right)$ and $H_{\mathfrak{a}}^{i}(M, N)=0$ for all $i>c$. Moreover, if $\operatorname{dim}\left(M \otimes_{R} N\right)<\infty$ and $d:=\operatorname{dim}\left(M \otimes_{R} N\right)+\operatorname{Gpd}_{N} M$, then $H_{\mathfrak{a}}^{d}(M, N)$ is Artinian and $\operatorname{Supp}_{R}\left(H_{\mathfrak{a}}^{d-1}(M, N)\right)$ is finite.

Proof. First of all, we claim that $H_{\mathfrak{a}}^{i}\left(\operatorname{Hom}_{R}(M, E)\right)=0$ for any injective $R$-module $E$ and all $i \geq 1$. Since any injective $R$-module decomposes into a direct sum of indecomposable injective $R$-modules, we may and do assume that $E=E_{R}(R / \mathfrak{p})$, for some prime ideal $\mathfrak{p}$ of $R$. (Note that the functor $H_{\mathfrak{a}}^{i}(\cdot)$ commutes with direct sums, and as $M$ is finitely generated the functor $\operatorname{Hom}_{R}(M, \cdot)$ also commutes with direct sums.) Since

$\operatorname{Hom}_{R}(M, E) \cong \operatorname{Hom}_{R}\left(M, \operatorname{Hom}_{R_{\mathfrak{p}}}\left(R_{\mathfrak{p}}, E\right)\right) \cong \operatorname{Hom}_{R_{\mathfrak{p}}}\left(M_{\mathfrak{p}}, E\right) \cong \operatorname{Hom}_{R_{\mathfrak{p}}}\left(M_{\mathfrak{p}}, E_{R_{\mathfrak{p}}}\left(R_{\mathfrak{p}} / \mathfrak{p} R_{\mathfrak{p}}\right)\right)$, we deduce that $\operatorname{Hom}_{R}(M, E)$ is an Artinian $R_{\mathfrak{p}}$-module. Hence $H_{\mathfrak{a}}^{i}\left(\operatorname{Hom}_{R}(M, E)\right)=$ $H_{\mathfrak{a} R_{\mathfrak{p}}}^{i}\left(\operatorname{Hom}_{R}(M, E)\right)=0$, as claimed. Let $F(\cdot):=\Gamma_{\mathfrak{a}}(\cdot)$ and $G(\cdot):=\operatorname{Hom}_{R}(M, \cdot)$. Since $(G F)(\cdot)=\operatorname{Hom}_{R}\left(M, \Gamma_{\mathfrak{a}}(\cdot)\right)$, by [즈. Theorem 11.38], one has the following Grothendieck's spectral sequence

$$
E_{2}^{i, j}:=H_{\mathfrak{a}}^{i}\left(\operatorname{Ext}_{R}^{j}(M, N)\right) \underset{i}{\Longrightarrow} H_{\mathfrak{a}}^{i+j}(M, N) .
$$

Hence for each $n \in \mathbb{N}_{0}$, there exists a chain

$$
0=H^{-1} \subseteq H^{0} \subseteq \cdots \subseteq H^{n}:=H_{\mathfrak{a}}^{n}(M, N) \quad(*)
$$

of submodules of $H_{\mathfrak{a}}^{n}(M, N)$ such that $H^{i} / H^{i-1} \cong E_{\infty}^{i, n-i}$ for all $i=0,1, \cdots, n$.

If $n>\operatorname{Gpd}_{N}(M)+\operatorname{cd}_{\mathfrak{a}}\left(M \otimes_{R} N\right)$, then either $i>\operatorname{cd}_{\mathfrak{a}}\left(M \otimes_{R} N\right)$ or $n-i>\operatorname{Gpd}_{N} M$. In each case, $E_{\infty}^{i, n-i}=0$, as $E_{\infty}^{i, n-i}$ is a subquotient of $E_{2}^{i, n-i}$. (Note that for each $j$, $\operatorname{cd}_{\mathfrak{a}}\left(\operatorname{Ext}_{R}^{j}(M, N)\right) \leq \operatorname{cd}_{\mathfrak{a}}\left(M \otimes_{R} N\right)$.) Therefore, from the chain $(*)$, it follows that $H_{\mathfrak{a}}^{n}(M, N)=0$ for all $n>\operatorname{Gpd}_{N}(M)+\operatorname{cd}_{\mathfrak{a}}\left(M \otimes_{R} N\right)$. 
Next, in the chain $(*)$, let $n=c$. Since $E_{2}^{i, j}=0$, whenever $i>c-p$ or $j>p$, it turns out that $H_{\mathfrak{a}}^{c}(M, N) \cong E_{\infty}^{c-p, p}$. For each $r \geq 2$, consider the sequence

$$
E_{r}^{c-p-r, p+r-1} \stackrel{d_{r}^{c-p-r, p+r-1}}{\longrightarrow} E_{r}^{\mathcal{c}-p, p} \stackrel{d_{r}^{c-p, p}}{\longrightarrow} E_{r}^{\mathcal{c}-p+r, p-r+1} .
$$

Since $E_{r}^{c-p-r, p+r-1}$ and $E_{r}^{c-p+r, p-r+1}$ are respectively subquotients of $E_{2}^{c-p-r, p+r-1}$ and $E_{2}^{c-p+r, p-r+1}$, it follows that $E_{r}^{c-p-r, p+r-1}=E_{r}^{c-p+r, p-r+1}=0$, and so

$$
E_{r+1}^{c-p, p}=\operatorname{ker} d_{r}^{c-p, p} / \operatorname{im} d_{r}^{c-p-r, p+r-1} \cong E_{r}^{c-p, p} .
$$

Hence $H_{\mathfrak{a}}^{c}(M, N) \cong E_{\infty}^{c-p, p} \cong \ldots \cong E_{2}^{c-p, p}$.

Now, assume that $\operatorname{dim}\left(M \otimes_{R} N\right)<\infty$ and set $d:=\operatorname{dim}\left(M \otimes_{R} N\right)+\operatorname{Gpd}_{N} M$. Clearly, $c \leq d$ and in view of the first assertion of the theorem, we may and do assume that $d=c$. As $H_{\mathfrak{a}}^{c}(M, N) \cong H_{\mathfrak{a}}^{c-p}\left(\operatorname{Ext}_{R}^{p}(M, N)\right)$ and $\operatorname{dim} \operatorname{Ext}_{R}^{p}(M, N) \leq c-p$, it follows that $H_{\mathfrak{a}}^{c}(M, N)$ is Artinian.

Let $i, j$ be two non-negative integers such that $i+j=d-1$. Lemma 2.4 yields that $\operatorname{dim} \operatorname{Ext}_{R}^{j}(M, N) \leq d-j$, and so $i=d-j-1 \geq \operatorname{dim} \operatorname{Ext}_{R}^{j}(M, N)-1$. So, by [Mar, Corollary 2.5], $\operatorname{Supp}_{R}\left(E_{\infty}^{i, j}\right)$ is finite. Thus, from the chain $(*)$, we deduce that $\operatorname{Supp}_{R}\left(H_{\mathfrak{a}}^{d-1}(M, N)\right)$ is finite.

In the above argument, we used the first assertion of Lemma 2.4. Slightly modifying it in the light of the second assertion of Lemma 2.4, concludes the following corollary. It is worth to mention that this corollary improves Theorem 3.1 and Lemma 5.4 in [CH]. It also generalizes [HZ, Theorem 3.2].

Corollary 2.6. Let $\mathfrak{a}$ be an ideal of $R$ and $M$ and $N$ two finitely generated $R$-modules. Assume that one of the following conditions is satisfied:

i) Gpd $M$ and pd $N$ are finite.

ii) id $M$ and Gid $N$ are finite.

iii) id $N$ is finite.

iv) $\operatorname{pd}_{N} M$ is finite.

Then $H_{\mathfrak{a}}^{i}(M, N)=0$ for all $i>\min \left\{\operatorname{dim} R, \operatorname{Gpd}_{N} M+\operatorname{cd}_{\mathfrak{a}}\left(M \otimes_{R} N\right)\right\}$. Moreover, if $\operatorname{dim}\left(M \otimes_{R} N\right)<\infty$ and $d:=\min \left\{\operatorname{dim} R, \operatorname{dim}\left(M \otimes_{R} N\right)+\operatorname{Gpd}_{N} M\right\}$, then $H_{\mathfrak{a}}^{d}(M, N)$ is Artinian and $\operatorname{Supp}_{R}\left(H_{\mathfrak{a}}^{d-1}(M, N)\right)$ is finite.

Among other things, Theorem 2.5 says that $\operatorname{cd}_{\mathfrak{a}}(M, N) \leq \operatorname{Gpd}_{N} M+\operatorname{cd}_{\mathfrak{a}}\left(M \otimes_{R} N\right)$. As the following corollary indicates, in some cases the equality holds.

Corollary 2.7. Let $\mathfrak{a}$ be an ideal of $R$ and $M$ and $N$ two finitely generated $R$-modules.

i) If $p:=\operatorname{Gpd}_{N} M=\operatorname{grade}\left(\operatorname{Ann}_{R} M, N\right)$, then $H_{\mathfrak{a}}^{i}(M, N)=H_{\mathfrak{a}}^{i-p}\left(\operatorname{Ext}_{R}^{p}(M, N)\right)$ for all $i$, and consequently $\operatorname{cd}_{\mathfrak{a}}(M, N)=\operatorname{Gpd}_{N} M+\operatorname{cd}_{\mathfrak{a}}\left(\operatorname{Ext}_{R}^{p}(M, N)\right)$.

ii) If $p:=\operatorname{pd}_{N} M=\operatorname{grade}\left(\operatorname{Ann}_{R} M, N\right)$, then $\operatorname{cd}_{\mathfrak{a}}(M, N)=\operatorname{Gpd}_{N} M+\operatorname{cd}_{\mathfrak{a}}\left(M \otimes_{R} N\right)$. 
iii) If $R$ is local, id $N<\infty$ and $M$ maximal Cohen Macaulay and faithful, then $\operatorname{cd}_{\mathfrak{a}}(M, N)=$ $\operatorname{cd}_{\mathfrak{a}}(N)$.

Proof. i) The spectral sequence $H_{\mathfrak{a}}^{i}\left(\operatorname{Ext}_{R}^{j}(M, N)\right) \Longrightarrow H_{\mathfrak{a}}^{i+j}(M, N)$ collapses at $j=p$, and so $H_{\mathfrak{a}}^{n}(M, N) \cong H_{\mathfrak{a}}^{n-p}\left(\operatorname{Ext}_{R}^{p}(M, N)\right)$ for all $n$. This shows that $\operatorname{cd}_{\mathfrak{a}}(M, N)=p+$ $\operatorname{cd}_{\mathfrak{a}}\left(\operatorname{Ext}_{R}^{p}(M, N)\right)$.

ii) Lemma 2.2 viii) yields that $\operatorname{cd}_{\mathfrak{a}}\left(\operatorname{Ext}_{R}^{p}(M, N)\right)=\operatorname{cd}_{\mathfrak{a}}\left(M \otimes_{R} N\right)$. Hence ii) follows by i).

iii) Lemma $2.2 \mathrm{ix}$ ) yields that $\operatorname{Gpd}_{N} M=0$, and so by the proof of i), we have the isomorphisms $H_{\mathfrak{a}}^{n}(M, N)=H_{\mathfrak{a}}^{n}\left(\operatorname{Hom}_{R}(M, N)\right)$ for all $n$. Since by [B̈B], Exercise 1.2.27], $\operatorname{Ass}_{R}\left(\operatorname{Hom}_{R}(M, N)\right)=\operatorname{Supp}_{R} M \cap \operatorname{Ass}_{R} N$, it follows that $\operatorname{Supp}_{R}\left(\operatorname{Hom}_{R}(M, N)\right)=$ $\operatorname{Supp}_{R} N$. Now, [DNT, Theorem 2.2] yield that

$$
\operatorname{cd}_{\mathfrak{a}}(M, N)=\operatorname{cd}_{\mathfrak{a}}\left(\operatorname{Hom}_{R}(M, N)\right)=\operatorname{cd}_{\mathfrak{a}}(N) .
$$

For two finitely generated $R$-modules $M$ and $N$, Theorem 2.5 implies that if $\operatorname{Gpd}_{N} M<$ $\infty$, then $\operatorname{cd}_{\mathfrak{a}}(M, N)<\infty$ for all ideals $\mathfrak{a}$ of $R$. The second part of the next corollary indicates that the converse is also true.

Corollary 2.8. Let $M$ and $N$ be two finitely generated $R$-modules.

i) Let $\mathfrak{a}$ be an ideal of $R$ such that $\operatorname{Supp}_{R} M \cap \operatorname{Supp}_{R} N \subseteq \mathrm{V}(\mathfrak{a})$. Then $H_{\mathfrak{a}}^{i}(M, N) \cong$ $\operatorname{Ext}_{R}^{i}(M, N)$ for all $i \in \mathbb{N}_{0}$.

ii) $\operatorname{Gpd}_{N} M<\infty$ if and only if $H_{\mathfrak{a}}^{i}(M, N)=0$ for all ideals $\mathfrak{a}$ of $R$ and for all $i \gg 0$.

Proof. i) Since

$$
\operatorname{Supp}_{R}\left(\operatorname{Ext}_{R}^{j}(M, N)\right) \subseteq \operatorname{Supp}_{R} M \cap \operatorname{Supp}_{R} N \subseteq \mathrm{V}(\mathfrak{a}),
$$

it follows that $\operatorname{Ext}_{R}^{j}(M, N)$ is a-torsion for all $i$. Hence, the spectral sequence

$$
H_{\mathfrak{a}}^{i}\left(\operatorname{Ext}_{R}^{j}(M, N)\right) \underset{i}{\Longrightarrow} H_{\mathfrak{a}}^{i+j}(M, N)
$$

collapses at $i=0$, and so

$$
H_{\mathfrak{a}}^{n}(M, N) \cong H_{\mathfrak{a}}^{0}\left(\operatorname{Ext}_{R}^{n}(M, N)\right)=\operatorname{Ext}_{R}^{n}(M, N)
$$

for all $n$.

ii) The "only if" part follows by Theorem 2.5. For the converse, let $\mathfrak{a}:=\operatorname{Ann}_{R}\left(M \otimes_{R}\right.$ $N)$. Then by i), we have $\operatorname{Ext}_{R}^{i}(M, N) \cong H_{\mathfrak{a}}^{i}(M, N)=0$ for all $i \gg 0$. Hence $\operatorname{Gpd}_{N} M<\infty$.

Sazeedeh [Sa1] has proved that local cohomology modules of an $R$-module $N$ can be computed by Gorenstein injective resolutions of $N$. Also, he [Sa2] proved that if $\operatorname{dim} R$ 
and pd $M$ are finite, then $H_{\mathfrak{a}}^{i}(M, N)$ can be computed by using Gorenstein injective resolutions of $N$. Next, by employing his technique that was used in the proof of [Sa1, Theorem 3.1], we improve his later-mentioned result.

Lemma 2.9. Let $\mathfrak{a}$ be an ideal of $R$ and $M$ a finitely generated $R$-module such that either $\operatorname{pd} M$ or id $M$ is finite. Then $H_{\mathfrak{a}}^{i}(M, N)=0$ for any Gorenstein injective $R$-module $N$ and all $i>0$. Hence for any $R$-module $N$, the generalized local cohomology modules $H_{\mathfrak{a}}^{i}(M, N)$ can be computed by Gorenstein injective resolutions of $N$.

Proof. Let $N$ be a Gorenstein injective $R$-module. Let $c:=\min \{\operatorname{pd} M, \operatorname{id} M\}+\operatorname{ara}(\mathfrak{a})$ and $E_{2}^{i, j}:=H_{\mathfrak{a}}^{i}\left(\operatorname{Ext}_{R}^{j}(M, N)\right)$ for all $i, j \geq 0$. If id $M<\infty$, then by Lemma $\left.2.2 \mathrm{vi}\right)$, $\operatorname{Gpd}_{N} M=0$. Therefore, the spectral sequence $E_{2}^{i, j} \Longrightarrow H_{\mathfrak{a}}^{i+j}(M, N)$ implies that $H_{\mathfrak{a}}^{i}(M, N)=0$ for all $i>c$ if either pd $M<\infty$ or id $M<\infty$. Since $N$ is Gorenstein injective, there exists an exact sequence

$$
I^{\bullet}: \cdots \longrightarrow I_{1} \longrightarrow I_{0} \longrightarrow I^{0} \longrightarrow I^{1} \longrightarrow \cdots
$$

of injective $R$-modules such that $N \cong \operatorname{ker}\left(I^{0} \longrightarrow I^{1}\right)$ and that $\operatorname{Hom}_{R}\left(I, I^{\bullet}\right)$ is exact for all injective $R$-modules $I$. For each $i>0$, let $N_{i}:=\operatorname{ker}\left(I_{i} \longrightarrow I_{i-1}\right), N_{0}:=\operatorname{ker}\left(I_{0} \longrightarrow I^{0}\right)$ and $N_{-1}:=N$. For each $i \geq 0$, from the exact sequence

$$
0 \longrightarrow N_{i} \longrightarrow I_{i} \longrightarrow N_{i-1} \longrightarrow 0,
$$

we deduce the following long exact sequences of generalized local cohomology modules

$$
\cdots \longrightarrow H_{\mathfrak{a}}^{j}\left(M, I_{i}\right) \longrightarrow H_{\mathfrak{a}}^{j}\left(M, N_{i-1}\right) \longrightarrow H_{\mathfrak{a}}^{j+1}\left(M, N_{i}\right) \longrightarrow H_{\mathfrak{a}}^{j+1}\left(M, I_{i}\right) \longrightarrow \cdots .
$$

Thus, we conclude the isomorphisms $H_{\mathfrak{a}}^{j}\left(M, N_{i-1}\right) \cong H_{\mathfrak{a}}^{j+1}\left(M, N_{i}\right)$ for all $i \geq 0$ and all $j \geq 1$. Hence for $i>0$, we have

$$
H_{\mathfrak{a}}^{i}(M, N) \cong H_{\mathfrak{a}}^{i+1}\left(M, N_{0}\right) \cong \ldots \cong H_{\mathfrak{a}}^{i+c}\left(M, N_{c-1}\right)=0 .
$$

(Note that $N_{c-1}$ is Gorenstein injective.) Thus any Gorenstein injective $R$-module is $H_{\mathfrak{a}}^{0}(M, \cdot)$-acyclic. This finishes the proof. Recall that if $T$ is a left exact additive functor from the category of $R$-modules and $R$-homomorphisms to itself, then for any $R$-module $N$, the right derived functors $R^{i} T$ of $T$ at $N$ can be computed by using right resolutions of $N$ which are consisting of T-acyclic modules.

Corollary 2.10. Let $\mathfrak{a}$ be an ideal of $R$ and $M, N$ finitely generated $R$-modules such that either $\operatorname{pd} M$ or id $M$ is finite. Then $\operatorname{cd}_{\mathfrak{a}}(M, N) \leq \min \left\{\operatorname{Gid} N, \operatorname{Gpd}_{N} M+\operatorname{cd}_{\mathfrak{a}}\left(M \otimes_{R} N\right)\right\}$.

Example 2.11. When Gpd $M$ is finite, it is rather natural to ask whether $H_{\mathfrak{a}}^{i}(M, N)$ can be computed by Gorenstein injective resolutions of $N$. This would not be the case. To see this, let $(R, \mathfrak{m}, k)$ be a Gorenstein local ring which is not regular. Then Gpd $k$ and Gid $k$ are both finite, while $H_{\mathfrak{m}}^{i}(k, k) \cong \operatorname{Ext}_{R}^{i}(k, k)$ is non-zero for infinitely many $i$. 


\section{THE LOCAL CASE}

In this section, for a pair $(M, N)$ of finitely generated modules over a Cohen-Macaulay local ring $(R, \mathfrak{m})$, we compute $\operatorname{cd}_{\mathfrak{m}}(M, N)$ provided that either $\mathrm{pd} M$ or id $N$ is finite. We will end this section by proving an analogue of the Hartshorne-Lichtenbaum Vanishing Theorem in the context of generalized local cohomology modules. The main ingredients in our proofs are Suzuki's and the Herzog-Zamani Duality Theorems for generalized local cohomology modules. We start this section with the following useful result.

Lemma 3.1. Let $\mathfrak{a}$ be an ideal of $R$ an $x$ an element of $R$. Let $M$ be a finitely generated $R$-module and $N$ an arbitrary $R$-module. There is a natural long exact sequence

$$
\cdots \longrightarrow H_{\mathfrak{a}+(x)}^{i}(M, N) \longrightarrow H_{\mathfrak{a}}^{i}(M, N) \longrightarrow H_{\mathfrak{a} R_{x}}^{i}\left(M_{x}, N_{x}\right) \longrightarrow H_{\mathfrak{a}+(x)}^{i+1}(M, N) \longrightarrow \cdots
$$

Proof. Let

$$
I^{\bullet}: 0 \longrightarrow I^{0} \stackrel{d^{0}}{\longrightarrow} I^{1} \longrightarrow \cdots \longrightarrow I^{i} \stackrel{d^{i}}{\longrightarrow} I^{i+1} \longrightarrow \cdots
$$

be an injective resolution of $N$. Since $M$ is finitely generated, [DST, Lemma $2.1 \mathrm{i})$ ] implies that $H_{\mathfrak{a}}^{i}(M, N) \cong H^{i}\left(\operatorname{Hom}_{R}\left(M, \Gamma_{\mathfrak{a}}\left(I^{\bullet}\right)\right)\right)$. Similarly, since the $R_{x}$-module $M_{x}$ is finitely generated and $I_{x}^{\bullet}$, the localization of $I^{\bullet}$ at $x$, provides an injective resolution for the $R_{x^{-}}$ module $N_{x}$, it turns out that $H_{\mathfrak{a} R_{x}}^{i}\left(M_{x}, N_{x}\right) \cong H^{i}\left(\operatorname{Hom}_{R_{x}}\left(M_{x}, \Gamma_{\mathfrak{a} R_{x}}\left(I_{x}^{\bullet}\right)\right)\right)$.

Let $I$ be an injective $R$-module. [BS, Lemma 8.1.1] yields the following split exact sequence

$$
0 \longrightarrow \Gamma_{\mathfrak{a}+(x)}(I) \stackrel{i}{\longrightarrow} \Gamma_{\mathfrak{a}}(I) \stackrel{f}{\longrightarrow} \Gamma_{\mathfrak{a}}\left(I_{x}\right) \longrightarrow 0,
$$

where the maps are the natural ones. Because of the natural isomorphism

$$
\operatorname{Hom}_{R_{x}}\left(M_{x}, \Gamma_{\mathfrak{a} R_{x}}\left(I_{x}\right)\right) \cong \operatorname{Hom}_{R}\left(M, \Gamma_{\mathfrak{a}}\left(I_{x}\right)\right),
$$

we can deduce the following exact sequence of complexes

$$
0 \longrightarrow \operatorname{Hom}_{R}\left(M, \Gamma_{\mathfrak{a}+(x)}\left(I^{\bullet}\right)\right) \stackrel{i^{\bullet}}{\longrightarrow} \operatorname{Hom}_{R}\left(M, \Gamma_{\mathfrak{a}}\left(I^{\bullet}\right)\right) \stackrel{f^{\bullet}}{\longrightarrow} \operatorname{Hom}_{R_{x}}\left(M_{x}, \Gamma_{\mathfrak{a} R_{x}}\left(I_{x}^{\bullet}\right)\right) \longrightarrow 0 .
$$

Its long exact sequence of cohomologies is precisely our desired long exact sequence.

Henceforth, we assume $(R, \mathfrak{m})$ is a local ring and $M, N$ finitely generated $R$-modules. We intend to compute $\operatorname{cd}_{\mathfrak{m}}(M, N)$. First, we specialize Theorem 2.5 and Lemma 2.6 to the case $\mathfrak{a}=\mathfrak{m}$.

Corollary 3.2. Let $(R, \mathfrak{m})$ be a local ring and $M$ and $N$ two finitely generated $R$-modules. Consider the following conditions:
i) $\operatorname{Gpd}_{N} M<\infty$.
ii) Gpd $M$ and $\mathrm{pd} N$ are finite.
iii) id $M$ and Gid $N$ are finite.
iv) id $N$ is finite. 
v) $\operatorname{pd}_{N} M$ is finite.

Put $d:=\operatorname{dim}\left(M \otimes_{R} N\right)+\operatorname{Gpd}_{N} M$ in case i) and $d:=\min \left\{\operatorname{dim} R, \operatorname{dim}\left(M \otimes_{R} N\right)+\right.$ $\left.\operatorname{Gpd}_{N} M\right\}$ in other cases. In each of the above cases, $\operatorname{cd}_{\mathfrak{m}}(M, N) \leq d$. Moreover for any ideal $\mathfrak{a}$ of $R$, in each of the above cases, $H_{\mathfrak{a}}^{d}(M, N)$ is a homomorphic image of $H_{\mathfrak{m}}^{d}(M, N)$.

Proof. By Grothendieck's non-Vanishing Theorem $\operatorname{cd}_{\mathfrak{m}}\left(M \otimes_{R} N\right)=\operatorname{dim}\left(M \otimes_{R} N\right)$. Hence, the first assertion is immediate by Theorem 2.5 and Corollary 2.6.

Now, we prove the second assertion. We may choose $x_{1}, x_{2}, \ldots, x_{n} \in R$ such that $\mathfrak{m}=$ $\mathfrak{a}+\left(x_{1}, x_{2}, \ldots, x_{n}\right)$. Set $\mathfrak{a}_{i}:=\mathfrak{a}+\left(x_{1}, \ldots, x_{i-1}\right)$ for $i=1, \ldots, n+1$. For each $1 \leq i \leq n$, by Lemma 3.1, we have the following long exact sequence of generalized local cohomology modules

$$
\cdots \longrightarrow H_{\mathfrak{a}_{i+1}}^{d}(M, N) \longrightarrow H_{\mathfrak{a}_{i}}^{d}(M, N) \longrightarrow H_{\mathfrak{a}_{i} R_{x_{i}}}^{d}\left(M_{x_{i}}, N_{x_{i}}\right) \longrightarrow \cdots
$$

By Theorem 2.5 or Corollary 2.6, $H_{\mathfrak{a}_{i}}^{d}(M, N)$ is Artinian. Hence $H_{\mathfrak{a}_{i}}^{d}(M, N)$ is supported at most at $\mathfrak{m}$, and so

$$
H_{\mathfrak{a}_{i} R_{x_{i}}}^{d}\left(M_{x_{i}}, N_{x_{i}}\right) \cong H_{\mathfrak{a}_{i}}^{d}(M, N)_{x_{i}}=0 .
$$

Hence the natural homomorphism $H_{\mathfrak{a}_{i+1}}^{d}(M, N) \longrightarrow H_{\mathfrak{a}_{i}}^{d}(M, N)$ is epic. Using this successively for $1 \leq i \leq n$, yields that $H_{\mathfrak{a}}^{d}(M, N)$ is a homomorphic image of $H_{\mathfrak{m}}^{d}(M, N)$.

The key to the proof of Theorem 3.5 below is given in the following lemma.

Lemma 3.3. Let $(R, \mathfrak{m}, k)$ be a Cohen Macaulay local ring and $M, N$ finitely generated $R$ modules such that pd $M<\infty$. Let $\omega_{\hat{R}}$ denote the canonical module of $\hat{R}$.

i) $\operatorname{grade}\left(\mathfrak{a} \hat{R}, \hat{M} \otimes_{\hat{R}} \omega_{\hat{R}}\right)=\operatorname{grade}(\mathfrak{a}, M)$ for any ideal $\mathfrak{a}$ of $R$.

ii) $\operatorname{Ass}_{R}\left(\hat{M} \otimes_{\hat{R}} \omega_{\hat{R}}\right)=\operatorname{Ass}_{R} M$.

iii) $\operatorname{Rad}\left(\operatorname{Ann}_{\hat{R}}\left(\operatorname{Hom}_{\hat{R}}\left(\omega_{\hat{R}}, \hat{N}\right)\right)\right)=\operatorname{Rad}\left(\operatorname{Ann}_{\hat{R}} \hat{N}\right)$. Moreover, if id $N<\infty$, then $\operatorname{Ann}_{\hat{R}}\left(\operatorname{Hom}_{\hat{R}}\left(\omega_{\hat{R}}, \hat{N}\right)\right)=\operatorname{Ann}_{\hat{R}} \hat{N}$.

Proof. i) We first prove the claim for $\mathfrak{a}=\mathfrak{m}$. Let $d:=\operatorname{dim} R$. Then, by Suzuki's Duality Theorem

$$
\operatorname{Ext}_{\hat{R}}^{i}\left(k, \hat{M} \otimes_{\hat{R}} \omega_{\hat{R}}\right) \cong H_{\mathfrak{m}}^{d-i}(M, k)^{\vee} \cong \operatorname{Ext}_{R}^{d-i}(M, k)^{\vee} .
$$

Hence, it follows by the Auslander-Buchsbaum formula that

$$
\begin{aligned}
\operatorname{depth}\left(\hat{M} \otimes_{\hat{R}} \omega_{\hat{R}}\right) & =\inf \left\{i: \operatorname{Ext}_{R}^{d-i}(M, k)^{\vee} \neq 0\right\} \\
& =\inf \left\{d-j: \operatorname{Ext}_{R}^{j}(M, k) \neq 0\right\} \\
& =d-\sup \left\{j: \operatorname{Ext}_{R}^{j}(M, k) \neq 0\right\} \\
& =\operatorname{depth} M .
\end{aligned}
$$

In particular, if $R$ possesses a canonical module $\omega_{R}$, then $\operatorname{depth} M=\operatorname{depth}\left(M \otimes_{R} \omega_{R}\right)$. 
Now, we prove the claim for an arbitrary ideal $\mathfrak{a}$. Without loss of generality, we may assume that $R$ is complete. It follows by $[\overline{\mathrm{BH}}$, Theorem 3.3.5 b)], that for each prime ideal $\mathfrak{p}$, the $R_{\mathfrak{p}}$-module $\left(\omega_{R}\right)_{\mathfrak{p}}$ is the canonical module of $R_{\mathfrak{p}}$. For any finitely generated $R$-module $L$, by [BH, Proposition 1.2.10 a)], we have $\operatorname{grade}(\mathfrak{a}, L)=\inf \left\{\operatorname{depth} L_{\mathfrak{p}}: \mathfrak{p} \in\right.$ $\mathrm{V}(\mathfrak{a})\}$. Thus

$$
\operatorname{grade}(\mathfrak{a}, M)=\inf \left\{\operatorname{depth}\left(M_{\mathfrak{p}} \otimes_{R_{\mathfrak{p}}}\left(\omega_{R}\right)_{\mathfrak{p}}\right): \mathfrak{p} \in \mathrm{V}(\mathfrak{a})\right\}=\operatorname{grade}\left(\mathfrak{a}, M \otimes_{R} \omega_{R}\right) .
$$

ii) Since the set $\operatorname{Ass}_{R}\left(\hat{M} \otimes_{\hat{R}} \omega_{\hat{R}}\right)$ (, respectively $\operatorname{Ass}_{R} M$ ) is precisely consisting of the contractions of elements of $\operatorname{Ass}_{\hat{R}}\left(\hat{M} \otimes_{\hat{R}} \omega_{\hat{R}}\right)$ (, respectively $\operatorname{Ass}_{\hat{R}} \hat{M}$ ) to $R$, we can assume that $R$ is complete. Remember that by the first paragraph of the proof, depth $M_{\mathfrak{p}}=$ $\operatorname{depth}\left(M \otimes_{R} \omega_{R}\right)_{\mathfrak{p}}$ for all prime ideal $\mathfrak{p}$ of $R$. But, for a finitely generated $R$-module $L$, one can check easily that $\mathfrak{p} \in \operatorname{Ass}_{R} L$ if and only if depth $L_{\mathfrak{p}}=0$. This yields that $\operatorname{Ass}_{R}\left(M \otimes_{R}\right.$ $\left.\omega_{R}\right)=\operatorname{Ass}_{R} M$.

iii) Since by [BH, Theorem 3.3.5 b)], Supp $\hat{R}_{\hat{R}} \omega_{\hat{R}}=\operatorname{Spec} \hat{R}$, it turns out by [BHE, Exercise 1.2.27] that

$$
\operatorname{Ass}_{\hat{R}}\left(\operatorname{Hom}_{\hat{R}}\left(\omega_{\hat{R}}, \hat{N}\right)\right)=\operatorname{Supp}_{\hat{R}} \omega_{\hat{R}} \cap \operatorname{Ass}_{\hat{R}} \hat{N}=\operatorname{Ass}_{\hat{R}} \hat{N} .
$$

Consequently, $\operatorname{Rad}\left(\operatorname{Ann}_{\hat{R}}\left(\operatorname{Hom}_{\hat{R}}\left(\omega_{\hat{R}}, \hat{N}\right)\right)\right)=\operatorname{Rad}\left(\operatorname{Ann}_{\hat{R}} \hat{N}\right)$, as required. If id $N<\infty$, then by [Su, Theorem 4.3 ii)], there is a natural isomorphism $\hat{N} \cong \operatorname{Hom}_{\hat{R}}\left(\omega_{\hat{R}}, \hat{N}\right) \otimes_{\hat{R}} \omega_{\hat{R}}$, which clearly implies that $\operatorname{Ann}_{\hat{R}}\left(\operatorname{Hom}_{\hat{R}}\left(\omega_{\hat{R}}, \hat{N}\right)\right)=\operatorname{Ann}_{\hat{R}} \hat{N}$.

The statement of the corollary below involves the notion of attached prime ideals. For convenient of the reader, we review this notion briefly in below. Let $A=A_{1}+\cdots+A_{n}$ be a minimal secondary representation of the Artinian $R$-module $A$. Then the ideals $\mathfrak{p}_{i}:=$ $\operatorname{Rad}\left(\operatorname{Ann}_{R} A_{i}\right)^{\prime}$ 's are prime and they are independent of the given minimal secondary representation. Each $\mathfrak{p}_{i}$ is said to be an attached prime ideal of $A$ and the set $\left\{\mathfrak{p}_{1}, \ldots, \mathfrak{p}_{n}\right\}$ is denoted by $\operatorname{Att}_{R} A$. It is easy to see that a prime ideal $\mathfrak{p}$ of $R$ is an attached prime ideal of $A$ if and only if there exists a quotient $C$ of $A$ such that $\mathfrak{p}=\operatorname{Ann}_{R} C$. In particular, this implies that $A$ is zero if and only if $\operatorname{Att}_{R} A$ is empty and that the set of attached prime ideals of any quotient of $A$ is contained in $\operatorname{Att}_{R} A$. Also over a complete local ring $(R, \mathfrak{m})$, by Matlis Duality Theorem and using this description of attached primes of $A$, it is straightforward to deduce the known fact that $\operatorname{Att}_{R} A=\operatorname{Ass}_{R}\left(\operatorname{Hom}_{R}\left(A, E_{R}(R / \mathfrak{m})\right)\right)$. For basic theory concerning attached prime ideals, we refer the reader to [Mat, Section 6, Appendix].

Corollary 3.4. Let $(R, \mathfrak{m})$ be a d-dimensional Cohen-Macaulay local ring and $M$ and $N$ two finitely generated $R$-modules such that either $\operatorname{pd} M$ or id $N$ is finite. Then

$$
\operatorname{Att}_{R}\left(H_{\mathfrak{m}}^{d}(M, N)\right)=\operatorname{Supp}_{R} N \cap \operatorname{Ass}_{R} M .
$$

In particular, $H_{\mathfrak{m}}^{d}(M, N)=0$ if and only if $\operatorname{Supp}_{R} N \cap \operatorname{Ass}_{R} M=\varnothing$. 
Proof. Let $f: R \longrightarrow T$ be a ring homomorphism and $L$ a $T$-module. Because for any $T$-module $K$, we have $\operatorname{Ann}_{R} K=f^{-1}\left(\operatorname{Ann}_{T} K\right)$, it follows that $\operatorname{Ass}_{R} L=\left\{f^{-1}(\mathfrak{p})\right.$ : $\left.\mathfrak{p} \in \operatorname{Ass}_{T} L\right\}$. Also by the same reason, if $L$ is Artinian as an $R$-module, then $\operatorname{Att}_{R} L=$ $\left\{f^{-1}(\mathfrak{p}): \mathfrak{p} \in \operatorname{Att}_{T} L\right\}$. Moreover, one can easily check that the $\operatorname{Supp}_{R} N$ is precisely consisting of the contractions to $R$ of the elements of $\operatorname{Supp}_{\hat{R}} \hat{N}$. Hence, we may assume that $R$ is complete.

First assume that pd $M<\infty$. Then, by Suzuki's Duality Theorem, $H_{\mathfrak{m}}^{d}(M, N) \cong$ $\operatorname{Hom}_{R}\left(N, M \otimes_{R} \omega_{R}\right)^{\vee}$. So, from [BH, Exercise 1.2.27] and Lemma 3.3 ii), it follows that

$$
\begin{aligned}
\operatorname{Att}_{R}\left(H_{\mathfrak{m}}^{d}(M, N)\right) & =\operatorname{Ass}_{R}\left(\operatorname{Hom}_{R}\left(N, M \otimes_{R} \omega_{R}\right)\right) \\
& =\operatorname{Supp}_{R} N \cap \operatorname{Ass}_{R}\left(M \otimes_{R} \omega_{R}\right) \\
& =\operatorname{Supp}_{R} N \cap \operatorname{Ass}_{R} M .
\end{aligned}
$$

Now, assume that id $N<\infty$. Then, by the Herzog-Zamani Duality Theorem,

$$
H_{\mathfrak{m}}^{d}(M, N)^{\vee} \cong \operatorname{Hom}_{R}\left(\operatorname{Hom}_{R}\left(\omega_{R}, N\right), M\right)
$$

Hence, from [BH, Exercise 1.2.27] and Lemma 3.3 iii), it follows that

$$
\begin{aligned}
\operatorname{Att}_{R}\left(H_{\mathfrak{m}}^{d}(M, N)\right) & \left.=\operatorname{Ass}_{R}\left(\operatorname{Hom}_{R}\left(\operatorname{Hom}_{R}\left(\omega_{R}, N\right), M\right)\right)\right) \\
& =\operatorname{Supp}_{R}\left(\operatorname{Hom}_{R}\left(\omega_{R}, N\right)\right) \cap \operatorname{Ass}_{R} M \\
& =\operatorname{Supp}_{R} N \cap \operatorname{Ass}_{R} M .
\end{aligned}
$$

Now, we are ready to present the first main result of this section.

Theorem 3.5. Let $(R, \mathfrak{m})$ be a Cohen-Macaulay local ring and $M$ and $N$ two finitely generated $R$-modules such that either $\mathrm{pd} M$ or id $N$ is finite. Then

$$
\operatorname{cd}_{\mathfrak{m}}(M, N)=\operatorname{dim} R-\operatorname{grade}\left(\operatorname{Ann}_{R} N, M\right) .
$$

Proof. Let $d:=\operatorname{dim} R$. First, assume that $\operatorname{pd} M<\infty$. Then by Suzuki's Duality Theorem, $H_{\mathfrak{m}}^{i}(M, N) \cong \operatorname{Ext}_{\hat{R}}^{d-i}\left(\hat{N}, \hat{M} \otimes_{\hat{R}} \omega_{\hat{R}}\right)^{\vee}$. Hence by [BH, Proposition 1.2.10 b)] and Lemma 3.3 i), it follows that

$$
\begin{aligned}
\operatorname{cd}_{\mathfrak{m}}(M, N) & =\sup \left\{i: \operatorname{Ext}_{\hat{R}}^{d-i}\left(\hat{N}, \hat{M} \otimes_{\hat{R}} \omega_{\hat{R}}\right)^{\vee} \neq 0\right\} \\
& =d-\inf \left\{j: \operatorname{Ext}_{\hat{R}}^{j}\left(\hat{N}, \hat{M} \otimes_{\hat{R}} \omega_{\hat{R}}\right) \neq 0\right\} \\
& =d-\operatorname{grade}\left(\left(\operatorname{Ann}_{R} N\right) \hat{R}, \hat{M} \otimes_{\hat{R}} \omega_{\hat{R}}\right) \\
& =d-\operatorname{grade}\left(\operatorname{Ann}_{R} N, M\right) .
\end{aligned}
$$

Now, assume that id $N<\infty$. Then by the Herzog-Zamani Duality Theorem

$$
H_{\mathfrak{m}}^{i}(M, N) \cong \operatorname{Ext}_{\hat{R}}^{d-i}\left(\operatorname{Hom}_{\hat{R}}\left(\omega_{\hat{R}}, \hat{N}\right), \hat{M}\right)^{\vee} .
$$

Therefore, in view of Lemma $3.3 \mathrm{iii}$ ), the assertion follows by repeating the above argument. 
Next, in the case $R$ is a Cohen-Macaulay local ring, we improve the dimension inequality $\operatorname{dim} N \leq \operatorname{pd} M+\operatorname{dim}\left(M \otimes_{R} N\right)$, which is one of the famous consequence of the New Intersection Theorem.

Corollary 3.6. Let $(R, \mathfrak{m})$ be a Cohen-Macaulay local ring and $M$ and $N$ two finitely generated $R$-modules. Assume that $\mathrm{pd} M$ is finite. Then

$$
\operatorname{dim} N \leq \operatorname{dim} R-\operatorname{grade}\left(\operatorname{Ann}_{R} N, M\right) \leq \operatorname{Gpd}_{N} M+\operatorname{dim}\left(M \otimes_{R} N\right) .
$$

Proof. By Theorem 3.5, $\mathrm{cd}_{\mathfrak{m}}(M, N)=\operatorname{dim} R-\operatorname{grade}\left(\operatorname{Ann}_{R} N, M\right)$, and so

$$
\operatorname{dim} R-\operatorname{grade}\left(\operatorname{Ann}_{R} N, M\right) \leq \operatorname{Gpd}_{N} M+\operatorname{dim}\left(M \otimes_{R} N\right),
$$

by parts iv) and v) of Corollary 3.2. On the other hand, since $\operatorname{pd} M$ is finite, any $M$ sequence is also an $R$-sequence. Hence $\operatorname{grade}\left(\operatorname{Ann}_{R} N, M\right) \leq \operatorname{grade}\left(\operatorname{Ann}_{R} N, R\right)=$ ht $\left(\operatorname{Ann}_{R} N\right)$, and so

$$
\operatorname{dim} N=\operatorname{dim} R-\operatorname{ht}\left(\operatorname{Ann}_{R} N\right) \leq \operatorname{dim} R-\operatorname{grade}\left(\operatorname{Ann}_{R} N, M\right)
$$

Example 3.7. In Theorem 3.5, the Cohen-Macaulayness assumption on $R$ is necessary. To see this, let $(R, \mathfrak{m})$ be a non Cohen-Macaulay local ring and $M, N$ finitely generated $R$ modules. Suppose that $M$ is Cohen-Macaulay and $\operatorname{pd} M<\infty$. Recall that the height of an ideal $\mathfrak{a}$ with respect to $M$ is defined by $h_{M} \mathfrak{a}:=\min \left\{\operatorname{dim} M_{\mathfrak{p}}: \mathfrak{p} \supseteq \mathfrak{a}\right\}$. We have

$$
\begin{aligned}
\operatorname{dim} R-\operatorname{grade}\left(\operatorname{Ann}_{R} N, M\right) & >\operatorname{depth} R-\operatorname{ht}_{M}\left(\operatorname{Ann}_{R} N\right) \\
& =\operatorname{pd} M+\operatorname{dim} M-\operatorname{ht}_{M}\left(\operatorname{Ann}_{R} N\right) \\
& =\operatorname{pd} M+\operatorname{dim}\left(M /\left(\operatorname{Ann}_{R} N\right) M\right) \\
& =\operatorname{Gpd}_{N} M+\operatorname{dim}\left(M \otimes_{R} N\right) .
\end{aligned}
$$

In the next result, we compute the kernel of the epimorphism $H_{\mathfrak{m}}^{d}(M, N) \longrightarrow H_{\mathfrak{a}}^{d}(M, N)$ in Corollary 3.2, when $d=\operatorname{dim} R$ and either pd $M$ or id $N$ is finite. Also, the following result is crucial for proving our analogue of the Hartshorne-Lichtenbaum Vanishing Theorem for generalized local cohomology modules. In what follows, for an Artinian $R$-module $A$, we put $\left\langle\mathfrak{m}>A:=\underset{i \in \mathbb{N}}{\cap} \mathfrak{m}^{i} A\right.$.

Theorem 3.8. Let $\mathfrak{a}$ be an ideal of a d-dimensional Cohen-Macaulay local ring $(R, \mathfrak{m})$ and $M$ and $N$ two finitely generated $R$-modules. If either $\operatorname{pd} M$ or id $N$ is finite, then there is a natural isomorphism

$$
H_{\mathfrak{a}}^{d}(M, N) \cong \frac{H_{\mathfrak{m}}^{d}(M, N)}{\sum_{i \in \mathbb{N}}<\mathfrak{m}>\left(0:_{H_{\mathfrak{m}}^{d}(M, N)} \mathfrak{a}^{n}\right)}
$$


Proof. We can assume that $R$ is complete. Let $\omega_{R}$ be the canonical module of $R$ and $(\cdot)^{\vee}:=\operatorname{Hom}_{R}\left(\cdot, E_{R}(R / \mathfrak{m})\right)$. Let $K$ and $L$ be finitely generated $R$-modules such that pd $L<\infty$ and set $A:=K^{\vee}$. Suzuki's Duality Theorem asserts that

$$
\operatorname{Ext}_{R}^{d}\left(K / \mathfrak{a}^{n} K, L \otimes_{R} \omega_{R}\right) \cong H_{\mathfrak{m}}^{0}\left(L, K / \mathfrak{a}^{n} K\right)^{\vee} .
$$

For a fixed integer $n \in \mathbb{N}$, choose an integer $t:=t(n) \in \mathbb{N}$ such that

$$
H_{\mathfrak{m}}^{0}\left(L, K / \mathfrak{a}^{n} K\right) \cong \operatorname{Hom}_{R}\left(R / \mathfrak{m}^{t}, \operatorname{Hom}_{R}\left(L, K / \mathfrak{a}^{n} K\right)\right) \cong \operatorname{Hom}_{R}\left(R / \mathfrak{m}^{t} \otimes_{R} L, K / \mathfrak{a}^{n} K\right)
$$

and $<\mathfrak{m}>\left(0::_{A} \mathfrak{a}^{n}\right)=\mathfrak{m}^{t}\left(0::_{A} \mathfrak{a}^{n}\right)$. It follows that

$$
\begin{aligned}
\operatorname{Ext}_{R}^{d}\left(K / \mathfrak{a}^{n} K, L \otimes_{R} \omega_{R}\right) & \cong\left(R / \mathfrak{m}^{t} \otimes_{R} L\right) \otimes_{R}\left(K / \mathfrak{a}^{n} K\right)^{\vee} \\
& \cong L \otimes_{R}\left(R / \mathfrak{m}^{t} \otimes_{R}\left(0:_{A} \mathfrak{a}^{n}\right)\right) \\
& \cong L \otimes_{R}\left(\frac{0: A \mathfrak{a}^{n}}{\left\langle\mathfrak{m}>\left(0:_{A} \mathfrak{a}^{n}\right)\right.}\right) .
\end{aligned}
$$

It is easy to check that $\underset{n}{\lim } \frac{0::_{A} \mathfrak{a}^{n}}{\left\langle\mathfrak{m}>\left(0:_{A} \mathfrak{a}^{n}\right)\right.}=\frac{A}{\sum_{n \in \mathbb{N}}<\mathfrak{m}>\left(0:_{A} \mathfrak{a}^{n}\right)}$. So [DS, Lemma 3.1] yields that

$$
H_{\mathfrak{a}}^{d}\left(K, L \otimes_{R} \omega_{R}\right) \cong L \otimes_{R} \frac{A}{\sum_{n \in \mathbb{N}}<\mathfrak{m}>\left(0:_{A} \mathfrak{a}^{n}\right)} \cong \frac{L \otimes_{R} K^{\vee}}{\sum_{n \in \mathbb{N}}<\mathfrak{m}>\left(0:_{L \otimes_{R} K^{\vee}} \mathfrak{a}^{n}\right)} .(*)
$$

Assume that id $N<\infty$. Then by [Su, Theorem $4.3 \mathrm{ii}$ ], there is a natural isomorphism $N \cong \operatorname{Hom}_{R}\left(\omega_{R}, N\right) \otimes_{R} \omega_{R}$. On the other hand,

$$
\operatorname{Hom}_{R}\left(\omega_{R}, N\right) \otimes_{R} M^{\vee} \cong \operatorname{Hom}_{R}\left(\operatorname{Hom}_{R}\left(\omega_{R}, N\right), M\right)^{\vee},
$$

and the later is isomorphic with $H_{\mathfrak{m}}^{d}(M, N)$, by the Herzog-Zamani Duality Theorem. Since, by [Su, Proposition 4.5 ii)], $\operatorname{pd}\left(\operatorname{Hom}_{R}\left(\omega_{R}, N\right)\right)<\infty$, from $(*)$, it follows that

$$
\begin{aligned}
H_{\mathfrak{a}}^{d}(M, N) & \cong H_{\mathfrak{a}}^{d}\left(M, \operatorname{Hom}_{R}\left(\omega_{R}, N\right) \otimes_{R} \omega_{R}\right) \\
& \cong \frac{H_{\mathfrak{m}}^{d}(M, N)}{\sum_{i \in \mathbb{N}}\left\langle\mathfrak{m}>\left(0_{H_{\mathfrak{m}}^{d}(M, N)} \mathfrak{a}^{n}\right)\right.} .
\end{aligned}
$$

Now, assume that pd $M<\infty$. By [Su, Proposition 4.5 i)], the functor $\cdot \otimes_{R} \omega_{R}$ is exact on the subcategory of modules of finite projective dimension, and consequently it follows that $\operatorname{Tor}_{i}^{R}\left(\omega_{R}, M\right)=0$ for all $i>0$. Hence, [CD, Lemma 2.5] and (*) imply that

$$
\begin{aligned}
H_{\mathfrak{a}}^{d}(M, R) & \cong H_{\mathfrak{a}}^{d}\left(M \otimes_{R} \omega_{R}, \omega_{R}\right) \\
& \cong \frac{\left(M \otimes_{R} \omega_{R}\right)^{\vee}}{\sum_{i \in \mathbb{N}}<\mathfrak{m}>\left(0:_{\left(M \otimes_{R} \omega_{R}\right)^{\vee \mathfrak{a}}}\right)} \\
& \cong \frac{H_{\mathfrak{m}}^{d}(M, R)}{\sum_{i \in \mathbb{N}}<\mathfrak{m}>\left(0_{H_{\mathfrak{m}}^{d}(M, R)^{\prime}}^{\left.\mathfrak{a}^{n}\right)}\right.} .
\end{aligned}
$$

Note that by Suzuki's Duality Theorem $\left(M \otimes_{R} \omega_{R}\right)^{\vee} \cong H_{\mathfrak{m}}^{d}(M, R)$. On the other hand, by Corollary $3.2 \mathrm{v})$, the functors $H_{\mathfrak{a}}^{d}(M, \cdot)$ and $H_{\mathfrak{m}}^{d}(M, \cdot)$ are right exact, and so using [DS, 
Lemma 3.1] once more, yields that

$$
\begin{aligned}
H_{\mathfrak{a}}^{d}(M, N) & \cong N \otimes_{R} \frac{H_{\mathfrak{m}}^{d}(M, R)}{\sum_{i \in \mathbb{N}}^{<\mathfrak{m}>\left(0:_{H_{\mathfrak{m}}^{d}(M, R)}\right.}} \\
& \cong \frac{\mathfrak{a}_{\mathfrak{m}}(M, N)}{\sum_{i \in \mathbb{N}}<\mathfrak{m}>\left(0:_{H_{\mathfrak{m}}^{d}(M, N)}^{\left.\mathfrak{a}^{n}\right)}\right.} .
\end{aligned}
$$

Corollary 3.9. Let $\mathfrak{a}$ be an ideal of a d-dimensional Cohen-Macaulay local ring $(R, \mathfrak{m})$ and $M$ and $N$ two finitely generated $R$-modules. If either $\mathrm{pd} M$ or id $N$ is finite, then

$$
\operatorname{Att}_{\hat{R}}\left(H_{\mathfrak{a}}^{d}(M, N)\right)=\left\{\mathfrak{p} \in \operatorname{Supp}_{\hat{R}} \hat{N} \cap \operatorname{Ass}_{\hat{R}} \hat{M}: \operatorname{dim}(\hat{R} / \mathfrak{a} \hat{R}+\mathfrak{p})=0\right\} .
$$

Proof. We can assume that $R$ is complete. Let $H_{\mathfrak{m}}^{d}(M, N)=A_{1}+\cdots+A_{n}$ be a minimal secondary representation of $H_{\mathfrak{m}}^{d}(M, N)$. We order the set $\operatorname{Att}_{R}\left(H_{\mathfrak{m}}^{d}(M, N)\right)=\left\{\mathfrak{p}_{1}, \ldots, \mathfrak{p}_{n}\right\}$ such that for an integer $0 \leq l \leq n, \operatorname{dim}\left(R / \mathfrak{a}+\mathfrak{p}_{i}\right)>0$ for all $1 \leq i \leq l$, while $\operatorname{dim}(R / \mathfrak{a}+$ $\left.\mathfrak{p}_{i}\right)=0$ for all $l+1 \leq i \leq n$. Then by [DS, Theorem 2.8], $A_{1}+\cdots+A_{l}$ is a minimal secondary representation of $B:=\sum_{n \in \mathbb{N}}<\mathfrak{m}>\left(0:_{H_{\mathfrak{m}}^{d}(M, N)} \mathfrak{a}^{n}\right)$. Now, it is easy to see that $\sum_{i=l+1}^{n}\left(A_{i}+B\right) / B$ is a minimal secondary representation of $H_{\mathfrak{a}}^{d}(M, N) \cong H_{\mathfrak{m}}^{d}(M, N) / B$. But $\left(A_{i}+B\right) / B \cong A_{i} /\left(A_{i} \cap B\right)$ is $\mathfrak{p}_{i}$-secondary for all $l+1 \leq i \leq n$. This finishes the proof by Corollary 3.4.

When $R$ is a Gorenstein local ring, an analogue of the Hartshorne-Lichtenbaum Vanishing Theorem for generalized local cohomology modules was established in [DST, Lemma 2.5]. Now, we weaken the assumption on $R$ to the Cohen-Macaulayness.

Corollary 3.10. Let $\mathfrak{a}$ be an ideal of a d-dimensional Cohen-Macaulay local ring $(R, \mathfrak{m})$ and $M$ and $N$ two finitely generated $R$-modules. Assume that either $\operatorname{pd} M$ or id $N$ is finite. Then the following are equivalent:

i) $H_{\mathfrak{a}}^{d}(M, N)=0$.

ii) $H_{\mathfrak{m}}^{d}(M, N)=\sum_{n \in \mathbb{N}}<\mathfrak{m}>\left(0:_{H_{\mathfrak{m}}^{d}(M, N)} \mathfrak{a}^{n}\right)$.

iii) For any integer $l \in \mathbb{N}$, there exists an $n=n(l) \in \mathbb{N}$ such that

$$
0:_{H_{\mathfrak{m}}^{d}(M, N)} \mathfrak{a}^{l} \subseteq<\mathfrak{m}>\left(0:_{H_{\mathfrak{m}}^{d}(M, N)} \mathfrak{a}^{n}\right) .
$$

iv) $\operatorname{dim} \hat{R} / \mathfrak{a} \hat{R}+\mathfrak{p}>0$ for all $\mathfrak{p} \in \operatorname{Supp}_{\hat{R}} \hat{N} \cap \operatorname{Ass}_{\hat{R}} \hat{M}$.

Proof. i), ii) and iv) are equivalent by Theorem 3.8 and Corollary 3.9, while the equivalence of ii) and iii) follows by [DS, Corollary 2.5].

\section{REFERENCES}

[AB] M. Auslander and M. Bridger, Stable module theory, Memoirs of the Amer. Math. Soc., 94, 1969.

[B] M.H. Bijan-Zadeh, A common generalization of local cohomology theories, Glasgow Math. J., 21(2), (1980), 173-181.

[BH] W. Bruns and J. Herzog, Cohen-Macaulay rings, Cambridge Studies in Advanced Math., 39, 1993. 
[BS] M. Brodmann and R.Y. Sharp, Local cohomology: an algebraic introduction with geometric applications, Cambridge Studies in Advanced Math., 60, 1998.

[CD] M. Chardin and K. Divaani-Aazar, A duality theorem for generalized local cohomology, Proc. Amer. Math. Soc., 136(8), (2008), 2749-2754.

$[\mathrm{CH}]$ N.Tu Cuong and N.Van Hoang, On the vanishing and the finiteness of supports of generalized local cohomology modules, Manuscripta Math., 126(1), (2008), 59-72.

[DNT] K. Divaani-Aazar, R. Naghipour and M. Tousi, Cohomological dimension of certain algebraic varieties, Proc. Amer. Math. Soc., 130(12), (2002), 3537-3544.

[DST] K. Divaani-Aazar, R. Sazeedeh and M. Tousi, On vanishing of generalized local cohomology modules, Algebra Colloq., 12(2), (2005), 213-218.

[DS] K. Divaani-Aazar and P. Schenzel, Ideal topologies, local cohomology and connectedness, Math. Proc. Cambridge Philos. Soc., 131(2), (2001), 211-226.

[EJ] E. Enochs and O.J. Jenda, Gorenstein injective and projective modules, Math. Z., 220(4), (1995), 611-633.

[He] J. Herzog, Komplex Auflösungen und Dualität in der lokalen Algebra, Habilitationsschrift, Universität Regensburg, 1974.

[HZ] J. Herzog and N. Zamani, Duality and vanishing of generalized local cohomology, Arch. Math. (Basel), 81(5), (2003), 512-519.

[Ho] H. Holm, Gorenstein homological dimensions, J. Pure Appl. Algebra, 189(1-3), (2004), 167-193.

[I] F. Ischebeck, Eine Dualität zwischen den Funktoren Ext und Tor, J. Algebra, 11(4), (1969) 510-531.

[JS] D.A. Jorgensen and L. Sega, Independence of the total reflexivity conditions for modules, Algebr. Represent. Theory 9(2), (2006), 217-226.

[KTY] L. Khatami, S. Yassemi and M. Tousi, Finiteness of Gorenstein injective dimension of modules, Proc. Amer. Math. Soc., 137(7), (2009), 2201-2207.

[Mar] T. Marley, The associated primes of local cohomology modules over rings of small dimension, Manuscripta Math., 104(4) (2001), 519-525.

[Mat] H. Matsumura, Commutative ring theory, Cambridge Studies in Advanced Math., 8, (1989).

[R] J. Rotman, An Introduction to Homological Algebra, Academic Press, San Diego, (1979).

[Sa1] R. Sazeedeh, Gorenstein injective modules and local cohomology, Proc. Amer. Math. Soc., 132(10), (2004), 2885-2891.

[Sa2] R. Sazeedeh, Gorenstein injective, Gorenstein flat modules and the section functor, J. Pure Appl. Algebra, 211(3), (2007), 773-783.

[Su] N. Suzuki, On the generalized local cohomology and its duality, J. Math. Kyoto. Univ., 18(1), (1978), 71-85.

[T] R. Takahashi, A characterization of modules locally of finite injective dimension, Proc. Amer. Math. Soc., 135(11), (2007) 3461-3464.

K. Divaani-Aazar, Department of Mathematics, Az-Zahra University, Vanak, Post Code 19834, TEHRAN, IRAN-AND-INSTITUTE FOR STUdiEs IN THEORETICAl PHySiCS AND MATHEMATICS, P.O. BOX 19395-5746, TEHRAN, IRAN.

E-mail address: kdivaani@ipm.ir

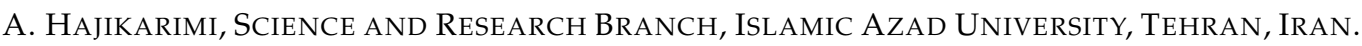

E-mail address: alihajikarimi@yahoo.com 